\title{
Variación espacial y temporal de la diversidad y abundancia de cianobacterias del Lago de Yojoa (2014-2015)
}

Alba Isbela Hernández Oviedo ${ }^{1}$

Mirna Marin ${ }^{2}$

\section{RESUMEN}

El objetivo del estudio fue identificar y analizar las variaciones temporales y espaciales de la diversidad y abundancia de las cianobacterias en el lago de Yojoa entre mayo 2014 y marzo 2015. Se analizaron 184 muestras identificandose 9 familias y 48 especies de cianobacterias, dominando Microcystis aeruginosa, Aphanocapsa delicatissima y Oscillatoria limosa.

El promedio de cel/ml de cianobacterias por muestreo disminuyeron progresivamente, el mayor 58,274 en muestreo1 y el menor 1,665 en muestreo 6. Es posible que la floración algal haya ocurrido días antes del muestreo 1, ya que se observó una nata superficial color café verdoso correspondiente a Oscillatoria limosa y Lyngbya sp. en proceso de descomposición, muchos fitoflagelados y vorticelas entre las colonias de Microcystis; coincidiendo con valores altos de nitrógeno y ortofosfato de ese muestreo.

La diversidad espacial y temporal fueron similares. La dominancia temporal fue mayor que la espacial, dominando: Microcystis aeruginosa, Chrococcus limneticus, Chrococcus sp, Aphanocapsa delicatissima, Aphanocapsa mucicola, Aphanocapsa elachista, Lyngbya sp, Gleocapsa sp y Pseudoanabaena mucicola. Se recomienda un plan de monitoreo y manejo de floraciones de cianobacterias tóxicas.

Palabras clave: Lago de Yojoa, fitoplancton, cianobacterias, Microcystis aeruginosa, Aphanacapsa delicatissima, Oscillatoria limosa.

\footnotetext{
${ }^{1}$ Profesor de Limnologia, Laboratorio Hidrobiología Escuela de Biologia, Facultad de Ciencias UNAH. albaoviedo2001@yahoo.com

${ }^{2}$ Asesora, Profesor Jubilado, Escuela de Biología, Facultad de Ciencias UNAH. namirmarin@gmail.com
} 


\section{ABSTRACT}

The objective of the study was to identify and analyze the temporal and spatial variations of the diversity and abundance of cyanobacteria in Lake Yojoa between May 2014 and March 2015. 184 samples were analyzed identifying 9 families and 48 species of cyanobacteria, dominating Microcystis aeruginosa, Aphanocapsa delicatissima and Oscillatoria limosa.

The average cell / $\mathrm{ml}$ of cyanobacteria per sample decreased progressively, the highest 58,274 in sampling 1 and the lowest in sampling 1,665. It is possible that the algal bloom occurred before days 1 , since a greenish-brown surface scum corresponding to Oscillatoria limosa and Lyngbya sp. in the process of decomposition, many phytoflagellates and vorticelles between the colonies of Microcystis; Coinciding with high nitrogen and orthophosphate values of that sample.

Spatial and temporal diversity were similar. The temporal dominance was greater than the spatial dominance: Microcystis aeruginosa, Chrococcus limneticus, Chrococcus sp, Aphanocapsa delicatissima, Aphanocapsa mucicola, Aphanocapsa elachista, Lyngbya sp, Gleocapsa sp and Pseudoanabaena mucicola were the dominant species. A monitoring plan and management of toxic cyanobacteria blooms is recommended.

Keywords: Lake Yojoa, phytoplankton, cyanobacteria, Microcystis aeruginosa, Aphanacapsa delicatissima, Oscillatoria limosa. 


\section{INTRODUCCIÓN}

Los cuerpos de agua continentales generalmente presentan un proceso de eutroficación natural, el que suele ser acelerado por acción antrópica. Una de las principales consecuencias de este proceso es el incremento de la densidad del fitoplancton, en especial de cianobacteria. Bonilla y Aubriot (2009) mencionan que: "Las cianobacterias son los organismos fotosintetizadores aeróbicos más antiguos del planeta. Las cianobacterias, bajo ciertas condiciones ambientales, tales como temperaturas elevadas, alta carga de nutrientes, especialmente (fosfatos y nitrógeno), baja relación N: P y $\mathrm{pH}$ alcalino desarrollan floraciones. Su capacidad de fijar nitrógeno atmosférico, la presencia de vesículas de gas, capacidad para crecer y reproducirse bajo distintas condiciones de luz calma ambiental y bajo caudal, entre otras, hace que resulte un grupo competitivamente exitoso con relación a otros del fitoplancton. Estas características posibilitan la formación de densas poblaciones que pueden se evidentes por acumulaciones en la superficie del agua, siendo detectables a simple vista y pudiendo producir un cambio de color en el agua. A estas proliferaciones se les conoce con el nombre de floraciones o blooms algales". Las floraciones suelen ser de ocurrencia estacional, especialmente durante los meses más cálidos del año o por procesos de eutroficación cultural, con sostenido aumento a nivel mundial; actualmente atribuido al cambio climático global (Pearl and Huisman, 2008, 2009), estas floraciones alteran el equilibrio del ecosistema acuático, impactando negativamente sobre la calidad del agua (Forastier y otros, 2013). A nivel ecológico lo más importante es la disminución de la biodiversidad y la falta de oxígeno, aunque en realidad estas son consecuencias directas de la presencia del afloramiento masivo independientemente de si éste es tóxico o no.

Sin embargo, la presencia de toxinas a nivel de ecosistema tiene gran importancia, porque puede suponer una variación en las especies de zooplancton, ya que muchas son sensibles a dichas toxinas, permaneciendo aquellas que no lo son, pero que pueden tener diferente valor nutricional para los peces que se alimentan de ellos 0 , incluso, pueden no servir de alimento para dichos peces, por lo que la estructura trófica del ecosistema quedaría desequilibrada. Además de estos problemas ecológicos, la presencia masiva de cianobacterias en nuestras aguas produce otra serie de problemas relacionados con el uso del recurso hídrico, como problemas estéticos que las hace perjudiciales para la recreación; problemas cuando el agua se utiliza para riego, porque obstruye válvulas y aspersores. En represas para consumo humano, el proceso de potalización se ve afectado porque se obstruyen y colmatan los filtros complicando el mismo. En muchos casos también confiere olores y sabores desagra- 
dables al agua mediante la producción de geosmina, que a pesar de no ser tóxica, inhabilita el uso del agua.

Las toxinas en los tejidos de algunos animales que las ingieren, invertebrados y vertebrados, pueden producir el proceso de biomagnificación, acumulando altas cantidades de toxinas circulando en la red trófica.

UNESCO (2009) menciona que la principal preocupación por las floraciones de cianobacterias se debe a que estos organismos pueden producir toxinas de cianobacterias denominadas cianotoxinas, que son compuestos químicos diversos agrupados dependiendo del modo en que actúan en el organismo: hepatotoxinas, neurotoxinas, saxitoxinas, nodularinas y anatoxinas (Chorus y Batram, 1999).

En ambientes altamente eutroficados se han producido en el mundo numerosas proliferaciones masivas de diferentes especies de cianobacterias. Además de los efectos negativos que se producen como consecuencia del crecimiento masivo de estas poblaciones, causan las alteraciones en la calidad del agua (en especial en el pH y oxígeno disuelto) y otras alteraciones indirectas por modificación de parámetros de toxicidad (fundamentalmente por el pH); actualmente se sabe que algunas especies producen toxinas potentes, capaces de producir efectos agudos y crónicos en el hombre, en animales y vegetales (Komárek, 1999; Roset y otros, 2001).

Las algas verde-azules, cianoficeas o cianobacterias, se han convertido en uno de los componentes más importantes del fitoplancton de los ecosistemas lénticos de Honduras, incluyendo embalses para agua potable. En las últimas décadas, en virtud de la intensa eutrofización de los ambientes acuáticos, las cianobacterias se han destacado principalmente por los problemas causados por sus floraciones, principalmente en las aguas destinadas para el abastecimiento público.

El conocimiento anterior y la progresiva eutroficación del lago de Yojoa, reportada por diferentes autores como Cruz y Delgado (1986), Sandoval (2003, 2005 ), Studer (2007) y Otero (2011), así como los alarmantes resultados de un muestreo realizado durante una práctica de laboratorio de limnología de la UNAH a finales de octubre de 2012, en el que fueron identificadas algunas especies de cianobacterias consideradas en la literatura como potencialmente tóxicas, que motivó a las autoras de este artículo a realizar un estudio puntual sobre la variación espacial y temporal de la diversidad y la abundancia del fitoplancton con énfasis en cianobacterias en el lago de Yojoa. 
Los procesos de eutrofización producen cambios cuantitativos y cualitativos en la comunidad de fitoplancton, aumentan la frecuencia y densidad de las floraciones y las cianobacterias desplazan las diatomeas, cloroficeas o crisofíceas. En ambientes altamente eutroficados suelen predominar durante la mayor parte del año. Estos hechos se han verificado en muchos trabajos limnológicos (Pizzolon, 1996), lo anterior fue verificado en un estudio reciente sobre la variación estpacial y temporal de la diversidad y abundancia del fitoplancton del lago de Yojoa en un año hidrológico 2014-2015 (Hernández y otros, 2016).

En el presente estudio se pretende examinar la importancia ecológica de la comunidad de cianobacterias del lago de Yojoa a través del análisis espacial y temporal de la diversidad y abundancia expresada como el número de cels/ml, durante un ciclo anual y su relación con las variables fisicoquímicas. Los resultados de la investigación serán muy importantes, ya que permitirán establecer programas de monitoreo y de alerta sanitaria temprana en los casos de afloramiento de cianobacterias potencialmente tóxicas a fin de apoyar a los pobladores en el control de casos de intoxicación producida por las mismas.

\section{MATERIALES Y METODOS}

La información correspondiente a ésta sección no se detalla en este artículo por cuanto la misma, está incorporada en el artículo "Variacion espacial y temporal de la diversidad y abundancia del fitoplancton del lago de Yojoa en un año hidrológico 2014-2015 "publicado en la Revista Ciencia y Tecnología No 19, diciembre del 2016 en las páginas 44-50.

\section{RESULTADOS Y DISCUSIÓN}

\section{Precipitación pluvial}

Los meses en los que se reportaron las mayores precipitaciones durante el estudio, correspondieron al tercer y cuarto muestreo durante la época lluviosa y al final de la época lluviosa e inicios de la época seca del 2014 respectivamente, las menores precipitaciones se registraron en época seca 2015 M5y M6 (Hernández y otros, 2016). 
Tabla 1. Promedios de los valores correspondientes los parámetros ambientales para cada muestreo durante el estudio.

\begin{tabular}{|c|c|c|c|c|c|c|c|}
\hline (ן/6u) оя০) & ח & 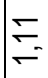 & $\mid \begin{array}{l}R \\
m\end{array}$ & $\underset{⿱ 亠 幺}{ \pm}$ & $\bar{\pi}$ & 每 & 尽 \\
\hline 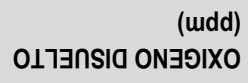 & 家 & 要 & 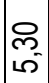 & \begin{tabular}{l}
8 \\
\multirow{8}{*}{}
\end{tabular} & 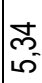 & $\underset{⿱ 亠 乂}{\mathbb{N}}$ & $\underset{\sim}{\mathscr{\gamma}}$ \\
\hline & $\dot{0}^{+}$ & $\underset{10}{N}$ & $\widetilde{N}$ & क् & $\infty$ & $\underset{f}{m}$ & 告 \\
\hline 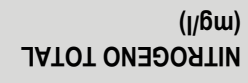 & 0 & $\hat{0}$ & $\dot{\sigma}_{-}^{-}$ & O্. & $\tilde{N}$ & $\approx$ & $\tilde{N}$ \\
\hline $\begin{array}{r}(\text { (//6u) } \\
7 \forall 10 \perp \text { OYOتSO }\end{array}$ & $\bar{\sigma}$ & $\widetilde{\sigma}$ & O. & $\tilde{O}^{\prime}$ & $\infty$ & $\widetilde{\sigma}$ & 第 \\
\hline 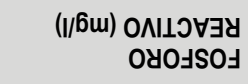 & 它 & 10 & $\widetilde{N}$ & tr & m & 120 & 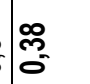 \\
\hline $\begin{array}{r}(/ / 6 m) \\
7 \forall \perp O \perp \forall Z \exists y \cap 0\end{array}$ & ㅇ. & ○. & $\stackrel{\dot{v}}{\boldsymbol{m}^{\prime}}$ & s & $\stackrel{\Omega}{\sim}$ & $\infty$ & $\mid \begin{array}{l}\infty \\
\infty \\
\sim\end{array}$ \\
\hline $\mathrm{Hd}^{\mathrm{d}}$ & $\bar{\infty}^{-}$ & م & $\bar{\infty}^{-}$ & $\infty$ & S & $\underset{\infty}{\sim}$ & 惢 \\
\hline 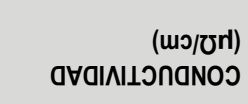 & @ & 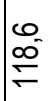 & $\stackrel{\substack{0 \\
\hdashline}}{\cong}$ & 훙 & ๙ึ & 足 & $\frac{1}{\varnothing}$ \\
\hline 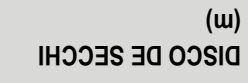 & $\hat{\sim}$ & $\stackrel{10}{\sim}$ & $\stackrel{\infty}{-}$ & $\underset{\sim}{\sim}$ & $\stackrel{m}{\sim}$ & $\begin{array}{l}0 \\
10\end{array}$ & $\mid \begin{array}{l}\infty \\
\sim \\
\sim\end{array}$ \\
\hline 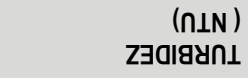 & $\stackrel{n}{8}$ & $\stackrel{0}{\circ}$ & $\underset{\sim}{\sim}$ & 吾 & $m$ & $\approx$ & $\mid \begin{array}{c}\infty \\
\stackrel{\infty}{\infty}\end{array}$ \\
\hline 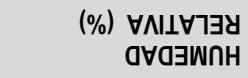 & $\begin{array}{l}m \\
0 \\
0\end{array}$ & 突 & $m$ & $\begin{array}{l}0 \\
\infty \\
\infty\end{array}$ & ○ & $\begin{array}{l}\sim \\
\sim 0 \\
10\end{array}$ & $\overrightarrow{8}$ \\
\hline 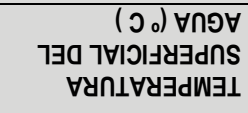 & $\stackrel{0}{\infty}$ & ন & $\begin{array}{l}\infty \\
\infty \\
\sim\end{array} \mid$ & L & $\ddot{N}$ & 足 & 官 \\
\hline 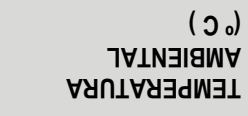 & חొ & ֻ̃ & $\begin{array}{l}m \\
0 \\
0\end{array}$ & $\stackrel{+}{\tilde{N}}$ & $\stackrel{\sim}{\sim}$ & 怘 & $\widehat{\hat{N}}$ \\
\hline 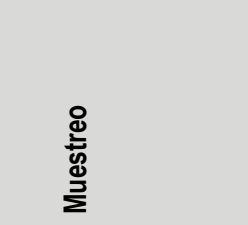 & 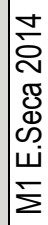 & 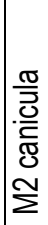 & 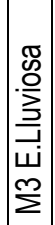 & 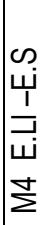 & 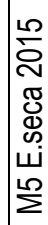 & 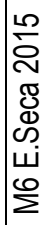 & 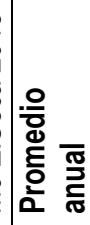 \\
\hline
\end{tabular}

Fuente: Elaborado con datos propios generados durante el estudio. 


\section{Variables ambientales}

Los promedios por cada muestreo y el anual, correspondientes a los parámetros ambientales se presentan en la Tabla 1.

El comportamiento de la temperatura del agua y el oxígeno disuelto en los primeros metros de profundidad del Lago pueden observarse en los perfiles de la Figura 1. El promedio de temperatura de $28^{\circ} \mathrm{C}$ del agua superficial de reportado para el Lago de Yojoa en los muestreos M1 (seca), M2 (canícula) y M3 (lluviosa) en ese orden y en todas las zonas monitoreadas fue relativamente alto.

Al observar los perfiles en la figura 1 se aprecia que la temperatura temporal y espacialmente se mantuvo relativamente constante desde la superficie hasta los $10 \mathrm{~m}$ de profundidad con pequeñas variaciones espaciales en el M1. En nuestro análisis no se aprecia estratificación térmica alguna como lo mencionan Goldman y Vaux (1984) y Sandoval (2003); a no ser que la estratificación se presente debajo de los $10 \mathrm{~m}$ de profundidad, medida no comprobada por limitaciones técnicas de la sonda con la que se trabajó. Sin embargo, en la figura 1 se observa que la temperatura comienza a disminuir a finales de la época lluviosa e inicio de la época seca (M4) y en la época seca (M5), lo que es coincidente con lo expresado por Sandoval (2003) que a partir de noviembre la temperatura del lago comienza a disminuir hasta el mes de enero, que es cuando se alcanzan las temperaturas más bajas en la superficie. Los perfiles de oxígeno y de temperatura muestran variaciones temporales y espaciales en la columna de agua. En M4 los perfiles son ligeramente heterogrados positivos, en M3 y M5 se observan perfiles ligeramente heterogrados negativoss. En M2 y M6 los perfiles se mantienen relativamente constantes desde la superficie hasta los $10 \mathrm{~m}$ de profundidad. En general, las curvas de la figura 1, temperatura versus oxígeno disuelto temporal y espacialmente desde la superficie hasta $10 \mathrm{~m}$ de profundidad, muestran ligeramente una relación inversamente proporcional.

Temporal y espacialmente las disminuciones de oxígeno disuelto desde metalimnio hasta hipolimnio se pueden deber al consumo por organismos heterotróficos a lo largo de la columna de agua y a la oxidación de materia orgánica en la capa del fondo, llevando al hipolimnio a un estado anóxico según la tendencia de dichos perfiles, de manera que las altas temperaturas superficiales permiten que se presenten altas tasas de descomposición en el hipolimnion, restringiendo a los organismos aeróbicos a vivir en estratos superficiales. En el hipolimnion se encuentran permanentemente productos metabólicos de desecho debido a las condiciones anóxicas imperantes (Horne y Goldman, 1994). 
Figura 1. Perfiles de oxígeno y temperatura en cada estación de muestreo por época de muestreo 2014-2015.

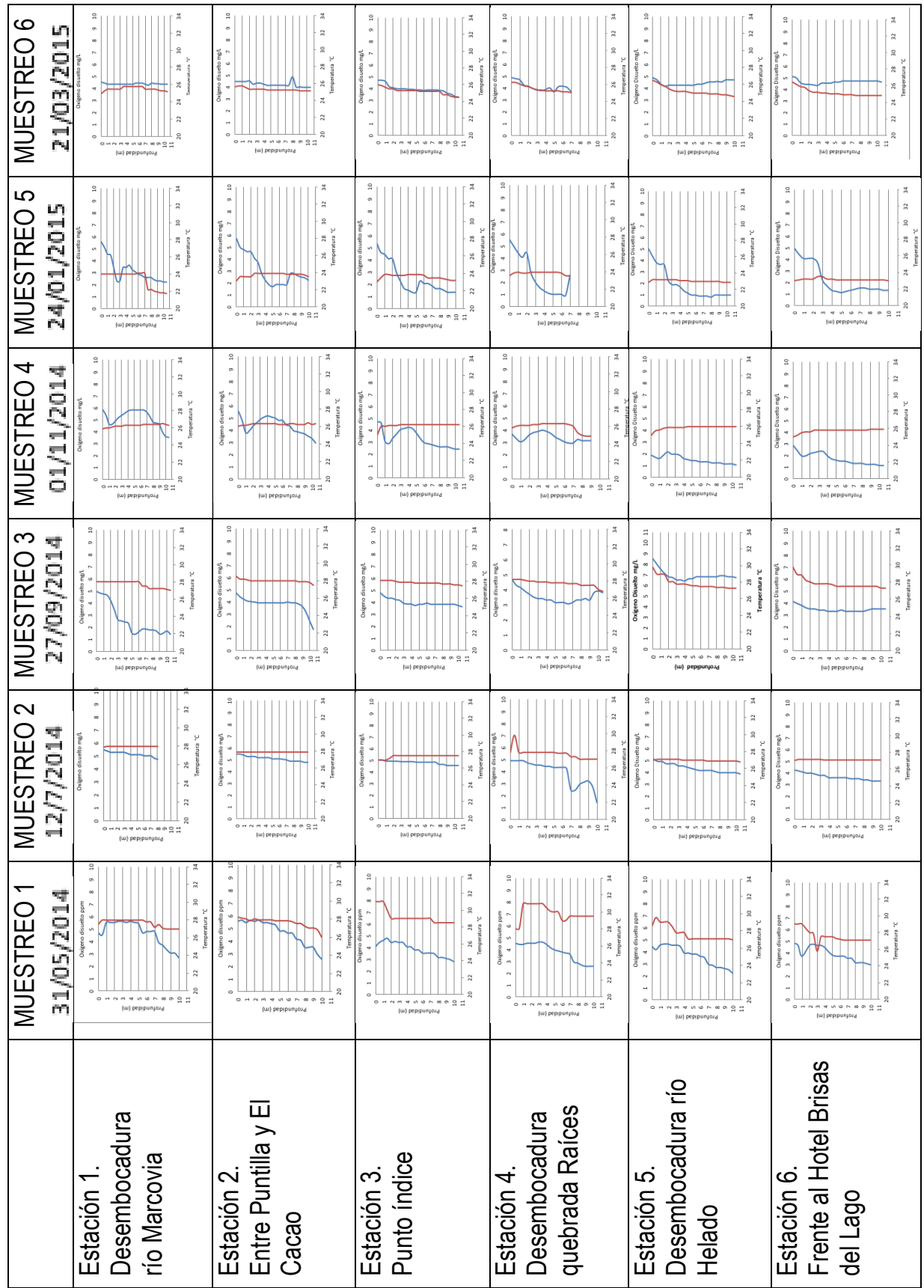

Perfiles de profundidad cada $50 \mathrm{~cm}$, mostrando curvas de oxígeno disuelto--- versus temperatura

Fuente: Elaboración propia a partir de los datos generados en la investigación. 
De los resultados obtenidos (Tabla 1) se puede inferir que el metabolismo del lago es atípico, debido a que constantemente tiene un flujo de descarga de agua controlado entre el epilimnio y el metalimnio, lo que hace que el comportamiento de las variables ambientales no sean coincidentes totalmente con lo expresado por Goldman y Vaux (1984) y Sandoval (2003). En ese sentido, se necesitan estudios a diferentes profundidades y sobre las corrientes internas del mismo.

En la Tabla 1, se observa que el promedio de oxígeno disuelto superficial más alto 5.34 ppm se registró en M5 y el promedio más bajo 4.06 ppm. en M4. Igualmente, en este estudio resultaron altos los valores promedios de DBO5 en M3 y M5 aunque también fueron altos; estos valores no son congruentes con lo esperado para un ecosistema natural, posiblemente los factores de la actividad antrópica y las variaciones estacionales pueden ser los responsables de esos valores pero se requieren estudios más detallados en ese tema.

Así mismo se puede observar que promedio de transparencia del agua en M6 la época seca (5.6 metros), donde se aprecia gran diferencia temporal con relación a los valores en M1-M5, diferencia que puede ser explicada por el aumento de las descargas para generación de energía relacionada con la precipitación alta en la subcuenca, debido a que los primeros metros son arrastrados por las corrientes y arrastran las capas biogénicas, situación que no coincide con lo mencionado por Wetzel (2001) en el que en un sistema léntico, los valores mayores de transparencia del agua se presentan durante la época de lluvias. En esta época la concentración de nutrientes se diluye por el aumento de caudales de los tributarios del lago, disminuyendo la turbidez de las aguas, la cual es básicamente de origen biogénico, característica típica de un sistema eutrófico.

Los valores promedios más altos de fósforo reactivo fueron en el M2 y en M6 y los de fósforo total en el M5; así como los valores promedios más altos de nitrógeno total en el M1 con leves variaciones espaciales. Los resultados anteriores demuestran que son coincidentes con la floración de cianobacterias en el (M1) y (M2), por lo que en el caso específico de los ortofosfatos en época lluviosa puede decirse que no fueron un factor limitante para el crecimiento de las cianobacterias.

Las concentraciones altas de estos nutrientes se deben a los aportes por las precipitaciones, escorrentías desde áreas agrícolas y aguas residuales tratadas insuficientemente, abonos y otros desechos de industrias de ganado, según lo señalado por Horne y Godman (1994); Melack (1996); Briand y otros (2003), así como por los desechos de cultivos de tilapia in situ. 


\section{Composición de la comunidad de cianobacterias en el lago de Yojoa}

El $59 \%$ de las especies del fitoplancton encontradas en el lago de Yojoa corresponden a la clase Cyanophyceae con 48 especies, el $22 \%$ a la clase Chlorophyceae, con 18 especies, 9 \% de la clase Bacyllariophyceae con 7 especies, el $4 \%$ la clase Zignematophyceae con 3 especies, el $3 \%$ la clase Euglenphyceae con 2 especies, el $2 \%$ a la clase Trebouxiophyceae con 2 especies y el $1 \%$ la clase Dinophyceae con 1 especie (Hernández y otros, 2016).

En la Tabla 2, se presentan las especies de cianobacterias encontradas en el lago de Yojoa durante todo el estudio, las cuales estuvieron compuestas por 21 géneros y 48 especies así; de las familias Nostocaceae (4 especies), Microcystaceae (6 especies), Chroococcaceae (5 especies), Synechoccaceae (12 especies), Oscillatoriaceae (10 especies), Phormidiaceae (3 especies), Borziaceae (1 especie), Pseudanabaenaceae (5 especies) y Merismopediaceae (2 especies).

\section{Variación espacial y temporal de las cianobacterias}

La variación espacial de la composición de la comunidad de cianobacterias (Tabla 3), se realizó con el análisis cualitativo a partir de las especies que tienen una frecuencia de aparición mayor al $40 \%$ como muy abundantes, entre $20 \%-40 \%$ abundantes, entre $19 \%-6 \%$ menos abundantes y raras entre $6 \%-3 \%$. Del total de 48 especies de cianobacterias determinadas en las seis estaciones monitoreadas durante los seis muestreos durante un año, 13 resultaron muy abundantes, 5 abundantes, 21 menos abundantes y 9 especies raras.

Espacial y temporalmente se registraron diferencias entre la composición taxonómica y la distribución de especies de cianobacterias entre los diferentes muestreos y estaciones muestreadas durante el estudio (Tabla 4) de acuerdo al análisis cualitativo, principalmente debido a la presencia, frecuencia, abundancia, dominancia y diversidad de los diferentes grupos encontrados. Las especies dominantes durante el estudio fueron Microcystis aeruginosa, Aphanocapsa delicatissima y Oscillatoria limosa (figura 2). 


\section{Tabla 2. Biodiversidad de cianobacterias en el lago de Yojoa, mayo de 2014 a} marzo de 2015

\begin{tabular}{|c|c|c|c|}
\hline CLASE & ORDEN & FAMILIA & GÉNERO \\
\hline \multirow[t]{27}{*}{ Cyanophyceae } & \multirow[t]{4}{*}{ Nostocales } & \multirow[t]{4}{*}{ Nostocaceae } & $\begin{array}{l}\text { Dolichospermum solitarium (Klebahn) } \\
\text { Wacklin, L.Hoffmann \& Komárek } 2009\end{array}$ \\
\hline & & & $\begin{array}{l}\text { Dolichospermum spiroides (Klebhan) } \\
\text { Wacklin, L.Hoffmann \& Komárek } 2009\end{array}$ \\
\hline & & & $\begin{array}{l}\text { Dolichospermum circinalis (Rabenhorst ex } \\
\text { Bornet \& Flahault) P.Wacklin, L.Hoffmann \& } \\
\text { J.Komárek } 2009\end{array}$ \\
\hline & & & $\begin{array}{l}\text { Dolichospermum sp. (Ralfs ex Bornet \& } \\
\text { Flahault) P.Wacklin, L.Hoffmann \& } \\
\text { J.Komárek, } 2009\end{array}$ \\
\hline & \multirow[t]{23}{*}{ Chrooccocales } & \multirow[t]{6}{*}{ Microcystaceae } & $\begin{array}{l}\text { Microcystis aeruginosa (Kützing) Kützing } \\
1846\end{array}$ \\
\hline & & & $\begin{array}{l}\text { Microcystis flosaquae (Wittrock) Kirchner } \\
1898\end{array}$ \\
\hline & & & Microcystis botrys Teiling 1942 \\
\hline & & & Microcystis potocystis W.B.Crow 1923 \\
\hline & & & Microcystis sp.Kützing, 1833 \\
\hline & & & Gleocapsa sp. Kützing, 1843 \\
\hline & & \multirow[t]{5}{*}{ Chroococcaceae } & Synechocystis sp C.Sauvageau, 1892 \\
\hline & & & Asterocapsa submersa Azevedo et al. 2003 \\
\hline & & & Chroococcus sp. Nägeli, 1849 \\
\hline & & & Chroococcus limneticus Lemmermann 1898 \\
\hline & & & Chroococcus dispersus Lemmermann 1904 \\
\hline & & \multirow[t]{12}{*}{ Synechoccaceae } & Aphanocapsa endophytica G.M.Smith 1920 \\
\hline & & & $\begin{array}{l}\text { Aphanocapsa delicatissima West \& } \\
\text { G.S.West } 1912\end{array}$ \\
\hline & & & $\begin{array}{l}\text { Aphanocapsa mucicola (Meneghini) Wille } \\
1919\end{array}$ \\
\hline & & & $\begin{array}{l}\text { Aphanocapsa holsatica (Lemmermann) } \\
\text { G.Cronberg \& Komárek } 1994\end{array}$ \\
\hline & & & Aphanocapsa koordesii Strom, 1923 \\
\hline & & & $\begin{array}{l}\text { Aphanocapsa planctonica (G.M.Smith) } \\
\text { Komárek \& Anagnostidis } 1995\end{array}$ \\
\hline & & & $\begin{array}{l}\text { Aphanocapsa incerta (Lemmermann) } \\
\text { G.Cronberg \& Komárek } 1994\end{array}$ \\
\hline & & & $\begin{array}{l}\text { Aphanocapsa elachista West \& G.S.West } \\
1894\end{array}$ \\
\hline & & & Aphanocapsa sp Nägeli, 1849 \\
\hline & & & Aphanothece sp Nägeli, 1849 \\
\hline & & & $\begin{array}{l}\text { Synechococcus nidulans } \\
\text { Komárek in Bourrelly } 1970\end{array}$ \\
\hline & & & Gloeothece sp. Bornet 1892 \\
\hline
\end{tabular}




\begin{tabular}{|c|c|c|}
\hline ORDEN & FAMILIA & GÉNERO \\
\hline \multirow[t]{13}{*}{ Oscillatoriales } & \multirow[t]{10}{*}{ Oscillatoriaceae } & Oscillatoria limosa C.Agardh ex Gomont 1892 \\
\hline & & Oscillatoria nigriviridis Thwaites ex Gomont 1892 \\
\hline & & Oscillatoria prínceps Vaucher ex Gomont 1892 \\
\hline & & Oscillatoria ornata Kützing ex Gomont 1892 \\
\hline & & Oscillatoria germinata Schwabe ex Gomont 1892 \\
\hline & & Oscillatoria sancta Kützing ex Gomont 1892 \\
\hline & & Oscillatoria sp. Vaucher ex Gomont, 1892 \\
\hline & & Lyngbya spirulinoidea Agardh ex Gomont, 1892 \\
\hline & & Lyngbya virgey C.Agardh ex Gomont, 1892 \\
\hline & & Lyngbya sp. C.Agardh ex Gomont, 1892 \\
\hline & \multirow[t]{3}{*}{ Phormidiaceae } & Phormidium sp. Kützing ex Gomont, 1892 \\
\hline & & Trichodesmium lacustre Klebahn 1895 \\
\hline & & Planktothrix sp. K.Anagnostidis \& J.Komárek, 1988 \\
\hline \multirow[t]{8}{*}{ Synechococales } & Borziaceae & Borzia trilocularis Cohn ex Gomont 1892 \\
\hline & \multirow[t]{5}{*}{ Pseudanabaenaceae } & $\begin{array}{l}\text { Leptolynghbya perelegans } \\
\text { Anagnostidis \& Komárek } 1988\end{array}$ \\
\hline & & Limnotrix sp Meffert, 1988 \\
\hline & & $\begin{array}{l}\text { Pseudanabaena mucicola (Naumann \& Huber- } \\
\text { Pestalozzi) Schwabe } 1964\end{array}$ \\
\hline & & Pseudanabaena galeata Böcher 1949 \\
\hline & & Pseudanabaena sp. Lauterborn, 1915 \\
\hline & \multirow[t]{2}{*}{ Merismopediaceae } & Coelosphaerium sp Nägeli, 1849 \\
\hline & & Limnococcus sp. Komárková et al. (2010) \\
\hline
\end{tabular}

\section{Figura 2. Fotografías de las especies de cianobacterias dominantes}

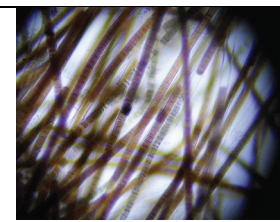

Oscillatoria limosa $10 \mathrm{X}$

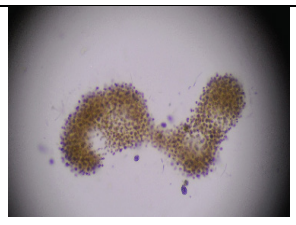

Microcystis aeruginosa $10 \mathrm{X}$

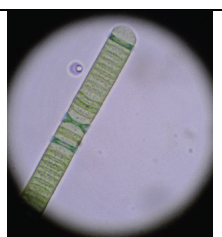

Oscillatoria limosa 40X

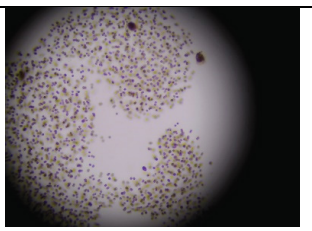

delicatissima $40 \mathrm{X}$

Fuente: Fotografias tomadas por Alba Isbela Hernández Oviedo. 
Es posible que la aparición de algunas especies esté determinada por las cianobacterias que se desarrollan en cada sistema lótico que aporta sus aguas al lago, convirtiéndose en un tema importante para abordar en otros estudios relativos a la comunidad fitoplanctónica , así mismo a las particularidades del lago como ser las corrientes prevalecientes de masas de agua con el manejo de las descargas para la Represa Cañaveral, corrientes internas, corrientes superficiales, etc. Es importante mencionar que otros factores como la morfología del lago, el clima y parámetros como luz y temperatura, $\mathrm{pH}$ y la forma y disponibilidad de los nutrientes para el fitoplancton, podrían haber influenciado la distribución de las cianobacterias en este ecosistema.

Se sabe que en lagos tropicales hay un patrón en la sucesión biológica por la hidrodinámica de la columna de agua y eventos meteorológicos (Hernández y otros, 2011), los cuales crean las condiciones ideales para el florecimiento de diferentes grupos algales. Así mismo, Reynolds (1988, 2006) y De León (2003), mencionan que en ambientes con limitación de nutrientes predominan organismos tolerantes al estrés como las cianofitas, de igual forma el flujo y disponibilidad de nutrientes son factores importantes que gobiernan la composición y biomasa de la comunidad fitoplanctónica en ecosistemas acuáticos, situación que es congruente con los resultados de este estudio en donde las cianobacterias predominaron durante los tres primeros muestreos (mayo, julio y septiembre) correspondiente a finales de la época seca e inicios de época lluviosa, veranillo (canícula) y en época lluviosa 2014, siendo mayor a finales de la época seca e inicio de la lluviosa y en el verano de 2015 cuando las temperaturas, la conductividad, promedios de ortofosfatos y de nitrógeno total y $\mathrm{pH}$ fueron altos, por lo que los factores físicos-químicos pudieron influenciar su distribución espacial y temporal.

Con el fin de comprender la variación espacial (estaciones) y temporal (muestreos) de las cianobacterias en el estudio, se realizó un análisis de las especies identificadas, en el cual se evidenció lo siguiente:

Estación 1. Localizada en la desembocadura del río Varsovia al sur del lago de Yojoa. De las 48 especies de cianobacterias identificadas, 28 estuvieron presentes. Dos especies, Dolichospermun spiroides en M3 y M4) y Oscilatoria germinata en M4 y M5, solo se encontraron en esta estación. Oscillatoria sancta en M4 y Lyngbya spirulinoidea en M1 se encontraron en E1 y E2, respectivamente.

Estación 2. Localizada en la desembocadura de la quebrada Jutiapa, entre E1 y E3. Aquí se encontraron 33 especies de cianobacterias de las 48 identificadas; de estas especies, solamente en E6 se hallaron más especies que las reportadas en esta 
estación. Además de las mismas especies de cianofitas encontradas también en la estación 1, las especies Oscilatoria sp. (M4) y Limnococcus sp. (M2) solamente fueron encontradas en esta estación.

Estación 3. Denominada Punto Índice (más o menos en el centro del lago), se reportaron 27 especies de cianobacterias de las 48 especies identificadas en todo el estudio. E3 presentó la mayor abundancia en el número de células por $\mathrm{ml}$, especialmente de Aphanocapsa delicatissima en el M2 y abundantes en M3, M5 y M6; de Microcystis aeruginosa en M4 a mediados de julio de 2014.

Estación 4. Localizada en la desembocadura de la quebrada Raíces, mejor conocida como la quebrada Cianuro. Aquí se encontraron 30 especies de cianobacterias de las 48 especies identificadas. Synechococcus nidulans (M2), Oscillatoria nigriviridis (M1) y Lyngbya virgey (M2) solamente se encontraron en esta estación. Sin embargo, Microcystis potocystis se encontró en esta estación y en la estación 5 , ambas en el M2. La mayor abundancia de células/ml. de Microcystis aeruginosa se encontró en esta estación en el M1 a finales de la época seca.

Estación 5. Denominada río Helado, se encuentra en la desembocadura del río Blanco, contiguo al canal de drenaje hacia la presa de El Cañaveral. Aquí se encontraron 31 especies de cianobacterias de las 48 identificadas en todo el estudio. Las especies encontradas corresponden a Microcystis flosaquae (M3), Asterocapsa submersa y Oscillaroria ornata (M1). Comparte la aparición de Microcystis botrys (M1) y Microcystis potocystis (M2) con las estaciones vecinas.

Estación 6. Localizada frente al Hotel Brisas del Lago. En esta estación se encontraron 33 especies de cianobacterias de las 48 identificadas en todo estudio. Las especies comunes con otras estaciones fueron Microcystis botrys con la estación 5 , siendo muy abundantes en M1 y Oscillatoria nigriviridis con la estación 4 en M4. De las especies de cianobacterias que solamente aparecieron en esta estación están: Planktothrix sp. (M2), Leptolyngbya perelegans (M2) y Limnotrix sp. (M1). 
Tabla 3. Variación de la frecuencia de especies de cianobacterias identificadas en el lago de Yojoa durante el estudio, mayo de 2014 a marzo de 2015

\begin{tabular}{|c|c|c|c|}
\hline Grupo & Especies & Frecuencia de aparición & Frecuencia relativa \\
\hline \multicolumn{4}{|c|}{ MUY ABUNDANTES } \\
\hline Cyano & Microcystis aeruginosa & 0.92 & 0.41 \\
\hline Cyano & Aphanocapsa delicatissima & 0.89 & 0.40 \\
\hline Cyano & Oscillatoria limosa & 0.89 & 0.40 \\
\hline Cyano & Aphanocapsa elachista & 0.72 & 0.33 \\
\hline Cyano & Pseudanabaena mucicola & 0.67 & 0.30 \\
\hline Cyano & Gleocapsa sp. & 0.64 & 0.29 \\
\hline Cyano & Aphanocapsa mucicola & 0.64 & 0.29 \\
\hline Cyano & Dolichospermum solitarium & 0.58 & 0.26 \\
\hline Cyano & Aphanocapsa sp. & 0.56 & 0.25 \\
\hline Cyano & Lyngbya sp. & 0.53 & 0.24 \\
\hline Cyano & Chrococcus sp. & 0.50 & 0.23 \\
\hline Cyano & Pseudanabaena galeata & 0.47 & 0.21 \\
\hline Cyano & Trichodesmium lacustre & 0.42 & 0.19 \\
\hline \multicolumn{4}{|c|}{ ABUNDANTES } \\
\hline Cyano & Microcystis sp. & 0.33 & 0.15 \\
\hline Cyano & Phormidium sp. & 0.31 & 0.14 \\
\hline Cyano & Aphanothece sp. & 0.28 & 0.13 \\
\hline Cyano & Aphanocapsa incerta & 0.25 & 0.11 \\
\hline Cyano & Aphanocapsa koordersii & 0.22 & 0.10 \\
\hline \multicolumn{4}{|c|}{ MENOS ABUNDANTES } \\
\hline Cyano & Borzia trilocularis & 0.19 & 0.09 \\
\hline Cyano & Oscillatoria prínceps & 0.19 & 0.09 \\
\hline Cyano & Chrococcus limneticus & 0.17 & 0.08 \\
\hline Cyano & Chrococcus dispersus & 0.17 & 0.08 \\
\hline Cyano & Aphanocapsa endophytica & 0.17 & 0.08 \\
\hline Cyano & Pseudanabaena sp. & 0.17 & 0.08 \\
\hline Cyano & Coelosphaerium sp. & 0.17 & 0.08 \\
\hline Cyano & Aphanocapsa holsatica & 0.14 & 0.06 \\
\hline Cyano & Dolichospermun circinalis & 0.14 & 0.06 \\
\hline Cyano & Dolichospermum sp. & 0.14 & 0.06 \\
\hline Cyano & Synechocystis sp. & 0.11 & 0.05 \\
\hline Cyano & Aphanocapsa planktonica & 0.11 & 0.05 \\
\hline Cyano & Dolichospermum spiroides & 0.08 & 0.04 \\
\hline Cyano & Microcystis botrys & 0.08 & 0.04 \\
\hline Cyano & Gloeothece sp. & 0.08 & 0.04 \\
\hline Cyano & Microcystis potocystis & 0.06 & 0.03 \\
\hline Cyano & Asterocapsa submersa & 0.06 & 0.03 \\
\hline Cyano & Oscillatoria nigriviridis & 0.06 & 0.03 \\
\hline Cyano & Oscillatoria germinata & 0.06 & 0.03 \\
\hline Cyano & Oscillatoria sancta & 0.06 & 0.03 \\
\hline Cyano & Lyngbya spirulinoidea & 0.06 & 0.03 \\
\hline \multicolumn{4}{|c|}{ RARAS } \\
\hline Cyano & Microcystis flosaquae & 0.03 & 0.01 \\
\hline Cyano & Oscillatoria sp. & 0.03 & 0.01 \\
\hline Cyano & Oscillatoria ornata & 0.03 & 0.01 \\
\hline Cyano & Lyngbya virgey & 0.03 & 0.01 \\
\hline Cyano & Synechococcus nidulans & 0.03 & 0.01 \\
\hline Cyano & Planktothrix sp & 0.03 & 0.01 \\
\hline Cyano & Leptolynghbya perelegans & 0.03 & 0.01 \\
\hline Cyano & Limnotrix sp. & 0.03 & 0.01 \\
\hline Cyano & Limnococcus sp. & 0.03 & 0.01 \\
\hline
\end{tabular}


Tabla 4. Variación espacial y temporal de cianobacterias en el lago de Yojoa durante el estudio, mayo de 2014-marzo de 2015

\begin{tabular}{|c|c|c|c|c|c|c|c|c|}
\hline $\begin{array}{l}\text { Especies muy abundantes en el } \\
\text { conteo de células }\end{array}$ & Especies & $\begin{array}{l}M \\
1\end{array}$ & \begin{tabular}{|l|}
$M$ \\
2
\end{tabular} & \begin{tabular}{l|}
$M$ \\
3
\end{tabular} & \begin{tabular}{|l|}
$M$ \\
4 \\
\end{tabular} & \begin{tabular}{|l|}
$M$ \\
5 \\
\end{tabular} & $\begin{array}{l}M \\
6\end{array}$ & Frec \% \\
\hline & Dolichospermum solitarium & 0 & 3 & 6 & 3 & 5 & 4 & 58 \\
\hline & Dolichospermun circinalis & 0 & 1 & 2 & 2 & 0 & 0 & 14 \\
\hline & Dolichospermum sp. & 0 & 0 & 2 & 1 & 1 & 0 & 11 \\
\hline & Dolichospermum spiroides & 0 & 1 & 1 & 1 & 0 & 0 & 8 \\
\hline \multirow[t]{7}{*}{ Muy abundantes en M1 y M4 } & Microcystis aeruginosa & 6 & 6 & 5 & 6 & 4 & 6 & 92 \\
\hline & Microcystis sp. & 3 & 2 & 1 & 2 & 0 & 4 & 33 \\
\hline & Microcystis botrys & 2 & 0 & 0 & 0 & 0 & 0 & 5,6 \\
\hline & Microcystis potocystis & 0 & 2 & 0 & 0 & 0 & 0 & 5,6 \\
\hline & Microcystis flosaquae & 0 & 0 & 1 & 0 & 0 & 0 & 2,7 \\
\hline & Gleocapsa sp. & 2 & 3 & 6 & 6 & 1 & 5 & 64 \\
\hline & Synechocystis sp. & 2 & 1 & 0 & 1 & 0 & 0 & 11 \\
\hline \multirow[t]{27}{*}{$\begin{array}{l}\text { Muy abundantes en M2, M3, M5 y } \\
\text { M6 }\end{array}$} & Aphanocapsa delicatissima & 2 & 6 & 6 & 6 & 6 & 6 & 89 \\
\hline & Aphanocapsa endophytica & 3 & 3 & 0 & 0 & 0 & 0 & 17 \\
\hline & Aphanocapsa elachista & 2 & 6 & 6 & 3 & 3 & 6 & 72 \\
\hline & Aphanocapsa mucicola & 3 & 4 & 4 & 3 & 3 & 6 & 64 \\
\hline & Aphanocapsa sp. & 2 & 2 & 4 & 4 & 5 & 3 & 56 \\
\hline & Aphanocapsa incerta & 0 & 0 & 4 & 1 & 3 & 1 & 25 \\
\hline & Aphanocapsa holsatica & 1 & 0 & 0 & 1 & 0 & 3 & 14 \\
\hline & Aphanocapsa koordersii & 1 & 0 & 0 & 1 & 0 & 3 & 14 \\
\hline & Aphanocapsa planctónica & 0 & 1 & 0 & 1 & 1 & 1 & 11 \\
\hline & Oscillatoria limosa & 6 & 6 & \begin{tabular}{|l|}
6 \\
\end{tabular} & 6 & 6 & 2 & 89 \\
\hline & Oscillatoria princeps & 1 & 0 & 2 & 2 & 2 & 0 & 19 \\
\hline & Oscillatoria nigriviridis & 1 & 0 & 0 & 1 & 0 & 0 & 6 \\
\hline & Oscillatoria germinata & 0 & 0 & 0 & 1 & 1 & 0 & 5,6 \\
\hline & Oscillatoria sancta & 0 & 0 & 0 & 1 & 1 & 0 & 5,6 \\
\hline & Oscillatoria ornata & 1 & 0 & 0 & 0 & 0 & 0 & 2,7 \\
\hline & Oscillatoria sp. & 0 & 0 & 0 & 1 & 0 & 0 & 2,7 \\
\hline & Lyngbya sp. & 0 & 1 & 3 & 3 & 6 & 6 & 53 \\
\hline & Lyngbya spirulinoidea & 2 & 0 & 0 & 0 & 0 & 0 & 5,6 \\
\hline & Lyngbya virgey & 0 & 1 & 0 & \begin{tabular}{|l|l|}
0 \\
\end{tabular} & 0 & 0 & 2,7 \\
\hline & Phormidium sp. & 2 & 3 & 3 & 2 & 1 & 1 & 33 \\
\hline & Planktothrix sp & 0 & 1 & 0 & 0 & 0 & 0 & 2,7 \\
\hline & Leptolynghbya perelegans & 0 & 1 & 0 & 0 & 0 & 0 & 2,7 \\
\hline & Pseudanabaena mucicola & 3 & 3 & 5 & 5 & 3 & 5 & 67 \\
\hline & Pseudanabaena galeata & 3 & 3 & 0 & 5 & 3 & 5 & 53 \\
\hline & Pseudanabaena sp. & 1 & 1 & 2 & 1 & 0 & 1 & 17 \\
\hline & Coelosphaerium sp. & 1 & 1 & 2 & 1 & 0 & 1 & 17 \\
\hline & $\begin{array}{l}\text { Especie } \\
\text { el contec }\end{array}$ & & & & & & & ciero \\
\hline
\end{tabular}

\section{Abundancia de la comunidad de cianobacterias}

De acuerdo con los resultados del análisis cuantitativo que se presentan en tabla 5 sobre la abundancia de la comunidad de cianobacterias, analizada en base al número de células/especie/ ml, resultó lo siguiente. 
En el M1 con 349,641 cel/ml de cianobacterias, de las cuales obtuvo 155,001 células/ml distribuidas en las estaciones E1 (río Varsovia), E2 (quebrada Jutiapa) y E3 (punto índice) y Microcystis sp con 192,466 cel/ml distribuidas en la E4 (quebrada Cianuro), E5 (río Helado) y E6 (Hotel Brisas del Lago); Oscillatoria limosa en las estaciones E1, E2 y E6 con $2001 \mathrm{cel} / \mathrm{ml}$. En el M2 con un total de 190,440 cel/ml de cianobacterias, de las cuales Aphanocapsa delicatissima con 188,361 cel/ml presentes en todas las estaciones, siendo más abundantes en la $\mathrm{E} 3$ con $82,000 \mathrm{cel} / \mathrm{ml}$, En el (M3) un total de 49,110 cel/ml, de éstas, 46,467 cel/ml corresponden a Aphanocapsa delicatissima, distribuidas en E1, E2, E3 y E6, siendo más abundante en E1 con $15,853 \mathrm{cel} / \mathrm{ml}$ y en E6 con 13,667 cel/ml y Aphanocapsa incerta con $1773 \mathrm{cel} / \mathrm{ml}$ en E1, E4, E5 y E6.

En el M4, un total de $23,519 \mathrm{cel} / \mathrm{ml}$, de las cuales $23,332 \mathrm{cel} / \mathrm{ml}$ corresponden a Microcystis sp. con presencia en todas las estaciones, siendo más abundantes en E6 con 6,667 cel/ml y Gleocapsa sp. con $187 \mathrm{cel} / \mathrm{ml}$ distribuidas en las estaciones E1, E2, E3, E4 y E6. En el M5 se reportaron 11,108 cel/ml, de las cuales 7,653 cel/ml corresponden a Aphanocapsa delicatissima distribuidas en E1, E2, E3, E4 y E5, siendo más abundantes en E5 con 2,187 cel/ml y Oscillatoria limosa con 2,001 cel/ml distribuidas en E1 con 1,334 cel/ml y en la E3 con $667 \mathrm{cel} / \mathrm{ml}$. Asimismo, estuvo presente Trichodesmium lacustre con 1,334 cel/ml en la E3 y E6. En el M6 aparecieron 9,991 cel/ml, siendo la especie más abundante Aphanocapsa delicatissima en la E1, E2, E4 y E5 con 5,834 cel/ml y Aphanocapsa elachista en E2, E4, E5 y E6 con 1,340 cel/ml y Lyngbya sp. en E2 con 2,000 cel/ml.

Con relación al promedio de cel/ml de cianobacterias por muestreo se puede observar en tabla 5 y figura 3 , que el número de células de cianobacterias/ml fue disminuyendo progresivamente durante el período de estudio, encontrándose el mayor promedio $(58,274)$ en el $\mathrm{M} 1$ al final de la época seca de 2014 y el menor número $(1,665)$ en el M6 de la época seca de 2015.

De acuerdo a los resultados del análisis cuantitativo (No. de células/ $\mathrm{ml}$ ) de las muestras del lago de Yojoa, se pudo determinar mayor abundancia en M1, M2 y M3 (Figura 3 y Tabla 5), siendo la mayor abundancia en M1, que coincidió con el final de un evento de floración; es posible que ésta haya ocurrido a mediados del mes de mayo de 2014, ya que en el M1 se observó una nata superficial de color café verdoso correspondiente a Oscillatoria limosa y Lyngbya sp. en proceso de descomposición. Igualmente, al momento de la identificación se observó abundancia de fitoflagelados y vorticelas entre las colonias de Microcystis aeruginosa. Este tipo de floración evidente en la superficie es de tipo acumulativa (Bonilla, S. y Aubriot, L, 2009). 
Figura 3. Variación del promedio por muestreo del biovolumen (número de células $/ \mathrm{ml}$ ) en las diferentes estaciones durante los diferentes muestreos en el lago de Yojoa del 30 de mayo de 2014 al 23 de marzo de 2015

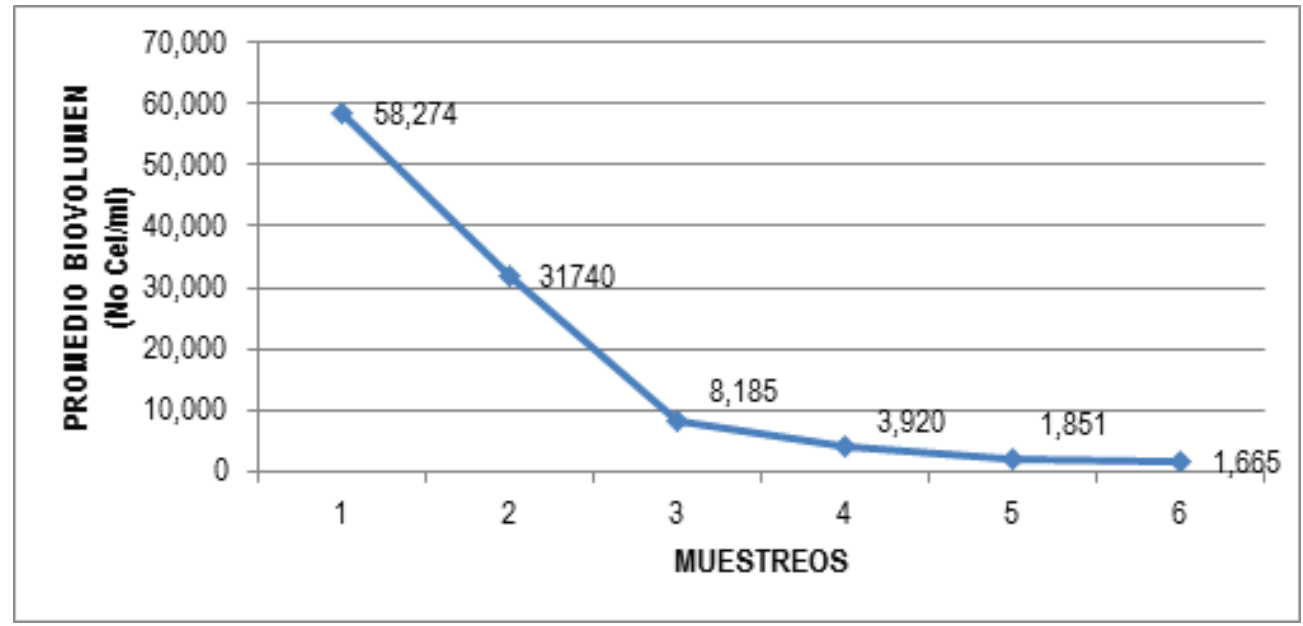

Fuente: Elaboración propia a partir de los datos generados en la investigación.

Tabla 5. Variación del número de células por $\mathrm{ml}$ (biovolumen) en el lago de Yojoa del 30 de mayo de 2014 al 23 de marzo de 2015.

\begin{tabular}{|l|l|l|l|l|l|l|}
\hline \multicolumn{6}{|l|}{ BIOVOLUMEN (No. de Cel/ml) } \\
\hline Estación & Muestreo 1 & Muestreo 2 & Muestreo 3 & Muestreo 4 & Muestreo 5 & Muestreo 6 \\
\hline E1 & 62334 & 31474 & 15913 & 3400 & 2974 & 1330 \\
\hline E2 & 57334 & 23523 & 10450 & 3373 & 1647 & 4766 \\
\hline E3 & 36834 & 82003 & 7650 & 3360 & 2427 & 217 \\
\hline E4 & 148333 & 21867 & 400 & 3373 & 1210 & 1224 \\
\hline E5 & 18336 & 18096 & 330 & 3333 & 2184 & 1554 \\
\hline E6 & 26467 & 11480 & 14367 & 6680 & 673 & 920 \\
\hline Total & 349641 & 190440 & 49110 & 23,519 & 11,108 & 9991 \\
\hline Prom & 58,274 & 31,740 & 8,185 & 3,920 & 1,851 & 1,665 \\
\hline Max & 148,333 & 82003 & 15,913 & 6,680 & 2974 & 4,766 \\
\hline Min & 26,467 & 11,480 & 330 & 3,333 & 673 & 920 \\
\hline
\end{tabular}

Fuente: Elaboración propia a partir de los datos generados en la investigación. 
Aunque no se observaron diferencias muy significativas en los resultados de algunos parámetros ambientales, es probable que los parámetros ambientales que estimularon el crecimiento de cianobacterias y de las variaciones temporales y espaciales en su densidad durante ese período fueron la radiación solar, las altas temperaturas, estabilidad de la columna de agua, promedios altos de ortofosfatos y de nitrógeno y pH ligeramente alcalino.

Es importante mencionar que el comportamiento del pH en el agua es el resultado de los iones suspendidos y de la productividad de las algas (Koschel, 1997). También, Pizzolon (1996) menciona que el pH elevado favorece el desarrollo de las cianobacterias por su capacidad para transformar los iones de bicarbonatos y carbonatos en dióxido de carbono. Cualquiera que sea el mecanismo involucrado, el predominio de las cianobacterias en muchos de los ecosistemas acuáticos es una respuesta del ambiente a la eutroficación cultural.

Es importante indicar que las estaciones en las que se observó mayor abundancia de células por $\mathrm{ml}$ de cianobacterias en el M1, corresponde a las estaciones E1 río Varsovia y E4 quebrada Raíces, las cuales reciben mayor aporte de nutrientes de origen antropogénico y por otra parte, la E3 Punto Indice en M2, posiblemente debido a los desechos producidos por el cultivo de tilapia.

A nivel mundial la frecuencia y duración de las floraciones de cianobacterias están en aumento. Esto ha sido atribuido al calentamiento global debido a que las cianobacterias crecen a temperaturas óptimas mucho más altas que las algas eucariotas. Así mismo, varios autores, basándose en observaciones de comunidades naturales en el laboratorio y en el campo, han destacado también el efecto positivo de la temperatura sobre el desarrollo de cianobacterias (Forastier y otros, 2013). Vela y otros (2007), indican que la temperatura y el $\mathrm{pH}$ influyen en las proliferaciones algales, siendo más propicias las temperaturas entre 20 y 30 grados, $\mathrm{pH}$ neutros o básicos. Forastier y otros (2013) demostraron experimentalmente que las cianobacterias pueden predominar con la presencia de rangos altos de nutrientes y a su vez, apuntan que el efecto de los nutrientes sobre el desarrollo de cianobacterias es menos significativo que la influencia que tienen los factores físicos, tales como temperatura, radiación solar y estabilidad de la columna de agua.

El promedio de las cianobacterias en el lago por muestreo superó las 20,000 células por ml encontradas en M1 y en M2 (Tabla 5). Los resultados anteriores hubieran justificado el establecimiento de alertas determinada por la OMS para cuerpos de agua de uso recreativo (Chorus y Bartram, 1999). Los resultados de este estudio 
ponen en evidencia la necesidad de que las organizaciones responsables del manejo del lago de Yojoa establezcan programas sistemáticos de alerta, seguimiento y monitoreo a los eventos de floraciones de cianobacterias, así como vigilar la aplicación de planes de manejo ya existentes.

Índices de diversidad, dominancia y similitud espacial y temporal de cianobacterias del lago de Yojoa durante el periodo de estudio

Espacialmente la diversidad de cianobacterias fue mayor 3.31 en la estación frente al Hotel Brisas del Lago (E6), posiblemente por el hecho de que esta estación se encuentra al final de la corriente predominante inducida hacia el canal de Cañaveral y la menor fue de 3.03 en E3 (punto índice o Aquafinca Saint Peter). Temporalmente, la diversidad fue mayor de 3.23 en M1 a finales del verano e inicio de la época lluviosa de 2014 y la menor fue de 2.96 en M5 a mediados del verano de 2015.

De acuerdo al resultado del índice de diversidad, es muy probable que las condiciones limnológicas del agua del lago pudieron haber influenciado espacial y temporalmente la diversidad de especies, y que éste resultado esté relacionado de manera general con el clima, especialmente la temperatura y con los nutrientes (Margalef, 1997). Por su parte, De León y Chalar (2003) mencionan que las variaciones de la diversidad están asociadas a las características productivas de la comunidad.

Espacialmente la dominancia durante el estudio, fue mayor (0.378) para la quebrada Raíces (E4) al final época lluviosa e inicio de la seca de 2014. Las especies dominantes en esta estación fueron: Microcystis aeruginosa, Aphanocapsa delicatissima, Aphanocapsa elachista, Aphanocapsa sp, Oscillatoria limosa, Pseudoanabaena mucícola y el valor menor fue de 0.247 en E3 (punto índice, Aquafinca Saint Peter Fish), con las especies: Microcystis aeruginosa, Aphanocapsa delicatissima, Oscillatoria limosa y Pseudoanabaena galeata. Temporalmente, la mayor dominancia fue de 0.586 en M6 en época seca, dominando las especies: Microcystis aeruginosa, Chrococcus limneticus, Chrococcus sp, Aphanocapsa delicatissima, Aphanocapsa mucicola, Aphanocapsa elachista, Lyngbya sp, Gleocapsa sp y Pseudoanabaena mucicola. El valor menor fue de 0.189 en M1 al final de la época seca. Las especies que dominaron fueron: Microcystis aeruginosa y Oscillatoria limosa.

De acuerdo a los resultados, no se observa variación significativa en la dominancia de especies de cianobacterias espacial y temporalmente entre estaciones y muestreos. Tanto espacial como temporalmente, los valores del índice de Berger y Parker resultaron bajos, siendo el M1 al final de la época seca e inicio de la lluviosa en la E3 
(punto índice, Aquafinca Saint Peter Fish), ambos resultados son indicadores de contaminación orgánica y degradación general y la especies identificadas son coincidentes con las especies dominantes indicadoras de sistemas de aguas continentales eutroficados.

Los valores de similitud, disimilitud o distancia entre estaciones (espacial), muestran que la mayor similitud fue de 0.73 entre E2 y E3 (quebrada Jutiapa y punto índice 0 Aquafinca Saint Peter) y la menor similitud fue de 0.47 entre E4 y E6, quebrada Raíces y frente al Hotel Brisas del Lago. La similitud entre muestreos (temporal) fue mayor (0.67) entre M3 y M5 en época lluviosa y a inicios de la época seca y entre M4 y M6 en la época lluviosa y época seca, respectivamente y la menor fue de 0.42 entre M2 y M5 a inicio de la época lluviosa e inicio de la época seca. De allí que al comparar los índices de similitud espacial, las estaciones vecinas E1-E2, E3-E4 y E5-E6 presentan índices relativamente altos. De igual forma, las estaciones que son influenciadas directamente por la desembocadura de cuerpos de agua corresponden a E1-E5, E2-E5 y E1-E6. De acuerdo a los resultados obtenidos, no hubo variación significativa, ya que tanto espacial como temporalmente se presentan especies en común. En todo el estudio, los valores de similitud espacialmente oscilaron entre 0.47 y 0.73 y temporalmente entre 0.42 y 0.67 .

De acuerdo al análisis de varianza (ANOVA), espacialmente el resultado correspondió a 0.63 y temporalmente a 0.78 . El promedio de organismos en el espacio y en el tiempo fue igual, con $95 \%$ de confiabilidad.

\section{CONCLUSIONES Y RECOMENDACIONES}

1. Aunque se han realizado muchos estudios en el lago de Yojoa, no se tiene información sobre las corrientes superficiales y de las masas de agua del mismo, ni tampoco existe un estudio sistemático de las especies de cianobacterias.

2. El $59 \%$ de las especies de fitoplancton encontradas en el lago de Yojoa corresponden a la clase Cyanophyceae con 48 especies de las familias Nostocaceae (4), Mycrocystaceae (6), Chroococcaceae (5), Synechoccaceae (12), Oscillatoriaceae (10), Phormidiaceae (3), Borziaceae (1), Psudoanabaenaceae (5) y Merimospediaceae (2).

3. Las especies dominantes durante el estudio fueron Microcystis aeruginosa, Aphanocapsa delicatissima y Oscillatoria limosa, especies potencialmente productoras de toxinas. 
4. Las cianobacterias predominaron durante los tres primeros muestreos con dominancia de Microcystis aeruginosa, Microcystis sp. y Oscilatoria limosa en mayo, correspondiente al final de la época seca e inicios de la lluviosa de 2014 con un promedio de 58,274 cel/ml. Aphanocapsa delicatissima y Oscillatoria limosa predominaron en julio correspondiente al veranillo o canícula con un promedio de $31,740 \mathrm{cel} / \mathrm{ml}$ y Aphanocapsa delicatissima, Aphanocapsa incerta y Aphanocapsa elachista en septiembre que corresponde a época lluviosa del 2014 con un promedio de $8,185 \mathrm{cel} / \mathrm{ml}$. , siendo mayor a finales de la época seca e inicio de la lluviosa de 2014 y en el verano de 2015 coincidiendo con temperaturas, conductividad, promedios de ortofosfatos y de nitrógeno total y $\mathrm{pH}$ altos, por lo que los factores físicos-químicos pudieron influenciar su distribución espacial y temporal.

5. Es posible que la aparición de especies reportadas solamente en algunas estaciones de muestreo esté determinada por las algas que se desarrollan en cada sistema lótico que aporta sus aguas al lago de Yojoa. Las variaciones espaciales y temporales de cianobacterias en el Lago de Yojoa además pudieron estar influenciadas por la morfología del lago, el clima, la disponibilidad de los nutrientes y especialmente las corrientes prevalecientes de las masas de agua relacionadas con el manejo de las descargas para la Represa Cañaveral. El patrón de lluvia en el lago pudo haber influido en el arrastre de cantidades considerables de fósforo y nitrógeno y otras sustancias que afectan el pH básico del agua superficial, turbidez y transparencia. Bajo ciertas condiciones, especialmente donde las aguas son ricas en nutrientes con aumento en el contenido de fósforo y nitrógeno provenientes principalmente de actividades agrícolas y de efluentes cloacales y alta radiación solar, las cianobacterias pueden multiplicarse hasta alcanzar altas densidades. Además, los parámetros como luz las temperaturas entre 20 y 30 grados y $\mathrm{pH}$ neutros o básicos son ideales para las floraciones de cianobacterias. Lo anterior se evidenció con la abundancia promedio de las cianobacterias en el primer muestreo (M1) correspondiente al final de la época seca e inicio de la época lluviosa, en la época lluviosa con presencia de veranillo(M2) y en la época lluviosa(M3) que coincidió con el final de un evento de floración algal.

6. A pesar del enriquecimiento por los nutrientes producido por las actividades acuícolas sin control y las actividades agrícolas (exceso de fertilizantes, aguas mieles y lavado de suelos por la deforestación y malas prácticas agropecuarias y agrícolas) en las microcuencas y en la zona litoral del lago, la corriente subsuperficial prevaleciente de sur a norte, contribuye a que el impacto no sea tan drástico para el metabolismo del sistema. Sin embargo, este enriquecimiento de nutrientes favorece a las cianobacterias, pudiendo haber floraciones de diferentes especies en cualquier época del año. 
7. Establecer un programa de monitoreo y manejo de florecimiento de algas toxicas. Lo cual requiere:

Muestreo, identificación y conteo permanente de especies indicadoras para un programa de alerta y manejo de los florecimientos algales y evitar problemas de salud de los pobladores y usuarios del area de influencia del Lago de Yojoa.

\section{AGRADECIMIENTOS}

A la Dirección de Investigación Científica de la Universidad Nacional Autónoma de Honduras por el soporte económico de una beca sustantiva para el desarrollo de ésta investigación. Asimismo, el agradecimiento a la Asociación de Municipios para la Protección del Lago de Yojoa (AMUPROLAGO) por el apoyo en infraestructura para la realización del trabajo de campo. Gracias también al doctor Oscar Parra y a la estudiante de doctorado Isis Yelena Montes, de la Universidad de Concepción, Chile por revisar y validar la clasificación de algunas de las cianobacterias. Igualmente se agradece a la Master Esperanza Izaguirre, Licenciados Marcela Garay, Ronald Castellón y Jorge Carranza por realizar los muestreos y al Departamento Técnico de la ENEE por habernos facilitado información hidrológica de 2014 y 2015 de la cuenca del lago de Yojoa.

\section{REFERENCIAS BIBLIOGRÁFICAS}

Bonilla, S. y Aubriot, L. (2009). Manual para la identificación y medidas de gestión. UNESCO. Briand, J.; Jacquet, S.; Bernard, C. \& Humbert, J. (2003). Health hazards for terrestrial vertebrales from toxic cyanobacteria in surface water ecosystems. Vertebrates Research, 34, 361-377.

Chorus, I. \& Bartram, J. (1999.) Tóxic cyanobacteria in water. A guide to their public Elath conseguenses, monitoring and managemente. London: W.H.O. E and FN spon.

Cruz, G. y Delgado, R. (1986). Distribución de macrófitas en el lago de Yojoa. Rev. Biol. Trop., 34(1), 141-149.

De León, L. y Guillermo Chalar. (2003). Abundancia y diversidad de fitoplancton en el embalse de Salto Grande (Argentina-Uruguay). Ciclo estacional y distribución espacial. Limnetica, 22(1-2), 103-113.

Forastier, M. E.; Zalocar, Y.; Sedan, D. y Andrinologo, D. (2013). Cyanobacteria y cianotoxinas en una laguna somera del Nordeste de Argentina. FACENA, 29, 65- 67.

Goldman, C. y Vaux, P. (1984). El Cajón hydroelectric proyect, limnology and fisheries 
program final report. Tegucigalpa: ENEE.

Hernández, M.R.; Ortega, M.R.; Sánchez, J.D.; Alvarado, V.R. y Aguilera, M.S. (2011). Distribución estacional del fitoplancton en un lago cálido monomíctico en Michoacán, México. Biológicas, 13(2), 21-28.

Hernandez, A.I.; Marin,M.; Herniquez ,M.L. y Garay. M. (2016). Variación espacial y temporal de la diversidad y abundancia del fitoplancton del Lago de Yojoa en un año hidrológico 2014-2015. Revista Ciencia y Tecnología, 19, 40-77.

Horne, A.J. y Goldman, C.R. (1994). Limnology. EE.UU.: McGraw Hill.

Koschel, R.H. (1997). Estructure and function of pelagic calcite precipitation in lake ecosystems. Verb. Int. Ver. Limnol, 26, 343-349.

Komárek, J. \& Anagnostidis, K. (1999). Cyanoprokaryota. I. Teil: Chroococales. Susswasserflora Von Mitteleuropa. Begr. Von Pascher Hrsg. Von H. Ette. Jena. G. Fischer Bd. 19. $548 \mathrm{pp}$.

Margalef, R. (1997). Excellence in Ecology. Germany: Ecology Institute.

Melack, J.M. (1996). Recent developments in tropical limnology. Verh. Internat. Verein. Limnol., 26, 211-217.

Otero, L. (2011). Temporalidad de parámetros de calidad en el lago de Yojoa, Honduras.

Tesis. Facultad de Geología, Universidad de Oviedo. España.

Pearl, H.W. \& Huisman, J. (2008). Blooms like it hot. Science, 320, 57-58.

Pearl, H.W. \& Huisman, J. (2009). Climate change: A catalyst for global expansiono f harmful cyanobacterial bloms. Environment Microbiology Reports, 1, 27-37.

Pizzolon, L. (1996). Importancia de las cianobacterias como factor de toxicidad en las aguas continentales. INTERCIENCIA, 21(6), 239-245.

Reynolds, C. S. (1988). Growth and reproductive strategies of freshwater phytoplankton. Cambridge. Inglaterra. Cambridge University Press.

Reynolds, C. S. (2006). Ecology of phytoplankton. Cambridge. Inglaterra. Cambridge University Press.

Roset, J.; Aguayo S. y Muñoz, M.J. (2001). Detección de cianobacterias y sus toxinas. Una revisión. Revista de Toxicología, 18, 65-71.

Sandoval, S. (2003). Evaluación de la calidad del agua del lago de Yojoa. Tegucigalpa: ENEE.

Sandoval, S. A. (2005). La verdad sobre la llamada contaminación de las aguas del lago de Yojoa. Ciencia y Tecnología, (15), 1-17.

Studer, E. (2007). Evaluación de parámetros físicos, químicos y biológicos. Indicadores del estado trófico del lago de Yojoa, Honduras. Tesis. EPFL-CESCCO.

UNESCO. (2009). Cianobacterias planctónicas del Uruguay. Manual para la identificación y medidas de gestión. Sylvia Bonilla (editora). Documento Técnico PH -LAC, N 16. Uruguay.

Vela, L.; Sevilla, E.; Martin, B.; Pellicer, S.; Bes, M.T.; Fillat, M.F. y Peleato, M.L. (2007). Las microcistinas. Rev. Real Academia de Ciencias, 62, 35-146. Zaragoza.

Wetzel, R.G. (2001). Limnology. EE.UU.: Academic Press. 